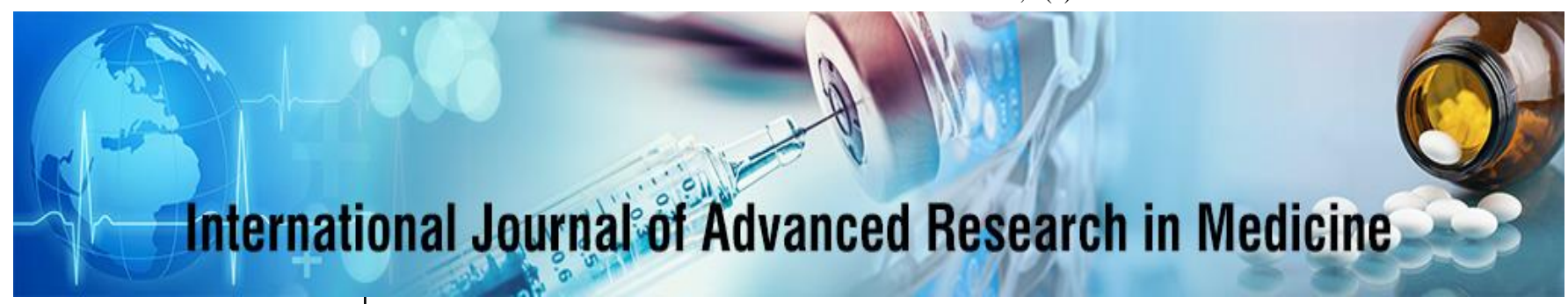

E-ISSN: 2706-9575

P-ISSN: 2706-9567

IJARM 2021; 3(2): 193-196

Received: 08-07-2021

Accepted: 09-08-2021

Dr. B Sreenivas Reddy

General Medicine, Gayatri

Hospital, Hasthinapuram,

Hyderabad, Telangana, India

Dr. Divya Reddy Molugu General Medicine, Gayatri Hospital, Hasthinapuram, Hyderabad, Telangana, India

Dr. Greeshma Reddy Molugu MBBS, Hyderabad,

Telangana, India
Corresponding Author: Dr. Divya Reddy Molugu General Medicine, Gayatri Hospital, Hasthinapuram, Hyderabad, Telangana, India

\section{Hyponatremia as a prognostic indicator in acute myocardial infarction}

\author{
Dr. Divya Reddy Molugu, Dr. B Sreenivas Reddy and Dr. Greeshma \\ Reddy Molugu
}

DOI: https://doi.org/10.22271/27069567.2021.v3.i2c.239

\begin{abstract}
Background: Every year, myocardial infarction causes more deaths and disabilities than any other disease in the world, and In fact, it is the most prevalent form of a life-threatening chronic illness.

Objectives: To study hyponatremia as a prognostic indicator in acute myocardial infarction

Methods: 100 patients with Acute Myocardial Infarction who were admitted to the ICU and the Department of Internal Medicine. All patients had a 12-lead ECG, with the right sided and posterior leads taken for inferior wall MI. A detailed history was collected, as well as a detailed clinical examination, as well as daily serum electrolytes and ECHO was done. An Ion selective electrode auto analyzer was used to determine the serum sodium concentration (Roche OMNIC).

Results: In our study $64 \%$ were males and $36 \%$ were females. Males are more prone for ischemic heart diseases. The proportion of Diabetic, Smokers, hypertensives were more among patients with Hyponatremia. anterior wall MI was the most prevalent, followed by inferior wall MI. Killip class I is found in $74 \%$ of patients with normal sodium levels compared to $58 \%$ in hyponatremia groups, class II in $20 \%$ of patients compared to $24 \%$ in hyponatremia, class III in $6 \%$ of patients compared to $16 \%$ of hyponatremia patients, and class IV in $2 \%$ of hyponatremia patients whose sodium level was 130 or lower. Patients who developed hyponatremia had a greater mortality rate than those who had normal sodium levels.

Conclusion: Asians are more likely to develop STEMI. The severe the Hyponatremia, the poorer the prognosis. Hyponatremia and a reduced ejection fraction are more common in anterior wall MI patients than those with normal sodium levels.
\end{abstract}

Keywords: Killip Score, STEMI, hyponatremia, myocardial infarction

\section{Introduction}

Coronary artery disease is a leading source of morbidity and mortality in both developing and developed countries. Cardiovascular disease is a significant cause of morbidity and death worldwide ${ }^{[1]}$. Every year, 3 million people die from sudden cardiac death as a result of an acute myocardial infarction. Cardiovascular disease has been labeled a contemporary epidemic by the World Health Organization (WHO) ${ }^{[2]}$. Acute Myocardial Infarction (MI), a symptom of coronary heart disease, is a medical emergency with physical, economic, and psychological consequences for humans ${ }^{[3]}$. With a decrease in infectious disease-related mortality due to faster economic growth and lifestyle changes that promote atherosclerosis, emerging nations, particularly India, are seeing a rise in ischemic heart disease.

Myocardial infarction is a condition in which there is insufficient blood and oxygen flow to a region of the myocardium; it generally develops when there is an imbalance between myocardial oxygen supply and demand. Because a myocardial infarction can occur at any time throughout a person's productive years, it can have serious negative, psychological, and economic consequences ${ }^{[4]}$. Atherosclerotic disease of an epicardial coronary artery is sufficient to produce a localized decrease in myocardial blood flow and insufficient perfusion of the myocardium supplied by the affected coronary artery is the most prevalent cause of myocardial ischemia. With a decrease in infectious disease-related mortality, rapid economic growth, and lifestyle changes that promote atherosclerosis, emerging nations, particularly India, are projected to see a rise in ischemic heart disease and Acute Myocardial Infractions (AMI) ${ }^{[5]}$. Given the vast discrepancy in available resources to treat AMI in poor countries, significant efforts are required to enhance community-based primary preventive programs. 
Obesity, insulin resistance, and type 2 diabetes mellitus are all on the rise, and they are all major risk factors for IHD and AMI.

Hyponatremia is a frequent electrolyte condition in hospitalized patients, particularly in those with heart failure, nephrotic syndrome, or cirrhosis. Hyponatremia has been proven to be a risk factor for cardiovascular disease in people with heart failure. Following a MI, hyponatremia is frequent, and clinical recovery is accompanied by an increase in plasma sodium content ${ }^{[6]}$.

However, whereas the predictive value of hyponatremia in chronic heart failure is well established in data, data on the prognostic value of hyponatremia in the setting of acute myocardial infarction are limited ${ }^{[7]}$. Hyponatremia is more common in the early stages of ST-elevation myocardial infarction and impacts both short and long-term outcomes.

\section{Materials and Methods}

Study Design: Retro prospective Observational study

Sample size: 100 patients with Acute Myocardial Infarction who were admitted to the ICU and the Department of Internal Medicine.

Patients were divided into two groups.

Group 1: (Control group): Sodium levels that are normal

Group 2: Sodium levels in the blood are less than $135 \mathrm{mEq} / \mathrm{L}$

\section{Observation and Results}

Table 1: Distribution based on variables

\begin{tabular}{|c|c|c|}
\hline Gender & Group 1 (Na>135) & Group 2 (Na<135) \\
\hline Male & $32(64 \%)$ & $32(64 \%)$ \\
\hline Female & $18(36 \%)$ & $18(36 \%)$ \\
\hline \multicolumn{3}{|c|}{ Age Group } \\
\hline$<40$ & $4(8 \%)$ & $4(8 \%)$ \\
\hline 41 to 50 & $8(16 \%)$ & $8(16 \%)$ \\
\hline 51 to 60 & $12(24 \%)$ & $22(44 \%)$ \\
\hline$>61$ & $26(52 \%)$ & $16(32 \%)$ \\
\hline Total & $50(100 \%)$ & $50(100 \%)$ \\
\hline \multicolumn{3}{|c|}{ Diabetes Mellitus } \\
\hline Yes & $25(50 \%)$ & $31(62 \%)$ \\
\hline No & $25(50 \%)$ & $19(38 \%)$ \\
\hline \multicolumn{3}{|c|}{ Hypertension } \\
\hline Yes & $23(46 \%)$ & $28(56 \%)$ \\
\hline No & $27(54 \%)$ & $22(44 \%)$ \\
\hline \multicolumn{3}{|c|}{$27(54 \%)$} \\
\hline Yes & $23(46 \%)$ & $22(44 \%)$ \\
\hline
\end{tabular}

In our study $64 \%$ were males and $36 \%$ were females. Males are more prone for ischemic heart diseases. In our study majority of cases were in age group of $>61$. Minimum age was 30, Maximum age was 80 .

In group 1: Among patients who had normal sodium levels $50 \%$ were Diabetic, $46 \%$ were Hypertensive, $54 \%$ were Smokers among patients who presented with Hyponatremia on admission. In Group 2: $62 \%$ were Diabetic, $56 \%$ were Hypertensive, $56 \%$ were smokers. The proportion of Diabetic, Smokers, hypertensives were more among patients with Hyponatremia.

\section{Inclusion Criteria}

- All cases of acute myocardial infarction in adults (STEMI)

\section{Exclusion Criteria}

- Infective endocarditis

- Any rheumatic heart disease history

- Cardiomyopathy

- Old MI

- Hypertensive patients on diuretics

Patients who met the following Inclusion and exclusion were included in the study.

All patients had a 12-lead ECG, with the right sided and posterior leads taken for inferior wall MI.

A detailed history was collected, as well as a detailed clinical examination, as well as daily serum electrolytes and ECHO was done.

An Ion selective electrode auto analyzer was used to determine the serum sodium concentration (Roche OMNIC). Hyponatremia is characterized as sodium levels below $135 \mathrm{mEq} / \mathrm{L}$.

Ethical Clearance: Ethical clearance was obtained from the institutional committee prior to the commencement of the study.

Statistical Methods: Statistical tables and charts were used to illustrate the statistical data. For statistical analysis SPSS software version 22 was used. 
Table 3: Distribution based on Ejection Fraction

\begin{tabular}{|c|c|c|c|}
\hline Ejection Fraction & Group 1 (Na>135) & Group 2 (Na<135) & Total \\
\hline$<50$ & 9 & 32 & 41 \\
\hline$>51$ & 41 & 18 & 59 \\
\hline
\end{tabular}

The Ejection Fraction was lower in patients who were diagnosed with hyponatremia or acquired hyponatremia within 72 hours when compared to individuals with normal sodium levels, which was statistically significant.

Table 4: Distribution based on KILLIP Classification

\begin{tabular}{|c|c|c|c|}
\hline KILLIP Class & Group 1 (Na>135) & Group 2 (Na<135) & Total \\
\hline I & 37 & 29 & 66 \\
\hline II & 10 & 12 & 22 \\
\hline III & 3 & 8 & 11 \\
\hline IV & 0 & 1 & 1 \\
\hline
\end{tabular}

Killip class I is found in $74 \%$ of patients with normal sodium levels compared to $58 \%$ in hyponatremia groups, class II in $20 \%$ of patients compared to $24 \%$ in hyponatremia, class III in $6 \%$ of patients compared to $16 \%$ of hyponatremia patients, and class IV in $2 \%$ of hyponatremia patients whose sodium level was 130 or lower.

Table 5: Distribution based on hospital complications

\begin{tabular}{|c|c|c|c|}
\hline $\begin{array}{c}\text { In hospital } \\
\text { complications }\end{array}$ & $\begin{array}{c}\text { Group 1 } \\
(\text { Na }>\text { 135 })\end{array}$ & $\begin{array}{c}\text { Group 2 } \\
(\text { Na< 135) }\end{array}$ & Total \\
\hline $2 *$ HB & 0 & 2 & 2 \\
\hline Acute MR & 1 & 1 & 2 \\
\hline CCF & 0 & 2 & 2 \\
\hline CHB & 0 & 2 & 2 \\
\hline PE & 2 & 1 & 3 \\
\hline Vfib & 0 & 1 & 1 \\
\hline VT & 0 & 1 & 1 \\
\hline Total & 3 & 10 & 13 \\
\hline
\end{tabular}

Patients with hyponatremia had a higher rate of hospital morbidity.

Table 6: Distribution based on Outcomes

\begin{tabular}{|c|c|c|c|}
\hline Outcome & Group 1 (Na>135) & Group 2 $(\mathbf{N a}<\mathbf{1 3 5})$ & Total \\
\hline Alive & 40 & 30 & 70 \\
\hline Death & 10 & 20 & 30 \\
\hline Total & 50 & 50 & 100 \\
\hline
\end{tabular}

Patients who developed hyponatremia had a greater mortality rate than those who had normal sodium levels.

\section{Discussion}

According to our results, individuals with acute myocardial infarction who had hyponatremia on admission or developed hyponatremia after admission are in a high risk category. In our study, a significant number of patients with acute myocardial infarction were hyponatremic at the time of admission or acquired hyponatremia shortly after admission. Patients with hyponatremia were more likely to have diabetes, an anterior infarction, a higher Killip class, and a lower ejection fraction. This is consistent with the findings of the Goldberg et al. research [9]. Klopotowski et al. observed that hyponatremia independently associated with in-hospital mortality in their research of 1858 ST-elevation
MI patients. Hyponatremic patients had a greater risk of inhospital mortality from a combination of death and heart failure ${ }^{[10]}$.

Our findings are consistent with those of Goldberg et al, who found that the degree of hyponatremia increased mortality. When we analyzed the different risk variables and outcomes among survivors and non-survivors, we observed that, in addition to age, gender, diabetes, hypertension, Killip class on admission, and ejection fraction, hyponatremia was a major risk factor in determining death. The multivariate logistic regression analysis included all of the factors that were substantially related with mortality among survivors and non-survivors. Hyponatremia was still a major independent predictor of mortality.

We conclude that the development of hyponatremia is a signal that most likely includes many prognostic variables, such as the severity of left ventricular failure, hemodynamic changes, and the level of neurohumoral activation ${ }^{[11]}$. It was shown that the development of hyponatremia is a biochemical marker for prognostic significance, i.e., the degree of left ventricular failure, hemodynamic alterations, and neurohumoral activation ${ }^{[12]}$. As a result, we found in our study that hyponatremia on admission or early development of hyponatremia in patients with acute MI is an independent predictor of 30-day mortality.

\section{Conclusion}

Asians are more likely to develop STEMI. The primary risk factors for acute myocardial infarction are smoking, diabetes, hypertension, and hypercholesterolemia. Hyponatremia that develops during admission or within 72 hours of admission has a poor prognosis. The severe the Hyponatremia, the poorer the prognosis. Hyponatremia and a reduced ejection fraction are more common in anterior wall MI patients than those with normal sodium levels.

\section{References}

1. Harrison principles of internal medicine, 20th edition, volume 2(269-270):1877-1890.

2. Basic and bedside Electrocardiography Romulo F. Baltazar 23:333-338 3.

3. Faxon DP, Williams DO: interventional cardiology: current status and future directions in coronary disease and valvular heart disease. circulation 2016;133:2697, 4.

4. Levine GN, et al: ACCF/AHA/SCAI Guideline for percutaneous coronary intervention: Executive Summary: A Report of the American College of Cardiology Foundation/ American Heart Association Task Force on Practice guideline and the society for cardiovascular Angiography and interventions. Circulation 2011;124:2574.

5. Vishram Singh. textbook of anatomy Vol 1, chapter 20, pericardium and heart page no 256, second edition 6 .

6. Robbins. and cotran pathologic basis of disease 9th edition chapter no.11 blood vessels page no. 491 and chapter no 12The Heart page no 5417.

7. Flear CT, Hilton P. Hyponatremia and severity and outcome of myocardial infarction. BMJ. 1979;1:12421246

8. Antman EM, Braunwald E. ST segment elevation mycocardial Infarction In: Zipes, Libby, Bonow, Braunwald editors. Braunwalds heart disease a text 
book of cardiovascular medicine.7th edition. Philadelphia: Elsevier Saunders 2005, 1141-1142. 9.

9. Goldberg A, Hammerman H, Petcherski S, Nassar M, Zdorovyak A, Yalonetsky S et al. Hyponatremia and long term mortality in survivors of acute ST elevation myocardial infarction. Arch Intern Med 2006;166:781786.

10. Klopotowski et al. Sodium level on admission and inhospital outcomes of STEMI patients treated with primary angioplasty: the ANIN Myocardial Infarction Registry; Med Sci Monit 2009;15:CR477-83.

11. Anderson RJ, Chung HM, Kluge R, Schrier RW. Hyponatremia: a prospective analysis of its epidemiology and the pathogenetic role of vasopressin. Ann Intern Med 1985;102(2):164-8

12. Singer GG, Brenner BM. Fluid and electrolyte disturbances. In: Kasper, Braunwald, Fauci, Hauser, Longo, and Jameson editors. Harrison's principles of Internal Medicine 1.20th edn. 298-301. 12. 